\title{
GENERALISED VARIATIONAL-LIKE INEQUALITIES AND A GAP FUNCTION
}

\author{
Q.H. ANSARI AND J.C. YaO
}

\begin{abstract}
In this paper, we study the existence of solutions of generalised variational-like inequality problems by using a generalised form of the Fan-KKM-Theorem. We also introduce a gap function for generalised variational-like inequalities.
\end{abstract}

\section{INTRODUCTION AND PRELIMINARIES}

Let $E$ be a topological vector space with dual $E^{*}$ and let $\left\langle E^{*}, E\right\rangle$ be the dual system of $E^{*}$ and $E$. We denote by $2^{X}$ the family of all nonempty subsets of a set $X$ and by $\mathcal{F}(X)$ the family of all nonempty finite subsets of $X$. If $X$ is a subset of a topological vector space $E$, we shall denote by $\bar{X}$ the closure of $X$ in $E$, and by $\operatorname{co}(\mathrm{X})$ the convex hull of $X$. Let $C$ and $K$ be nonempty subsets of $E$ and $E^{*}$, respectively. Given two maps $\theta: C \times K \rightarrow E^{*}$ and $\eta: C \times C \rightarrow E$, and a multifunction $T: C \rightarrow 2^{K}$, then we consider the following generalised variational-like inequality problems:

Problem 1. Find $\bar{x} \in C$ and $\bar{s} \in T(\bar{x})$ such that

$$
\langle\theta(\bar{x}, \bar{s}), \eta(\bar{x}, y)\rangle \leqslant 0, \quad \text { for all } \quad y \in C
$$

The vector $\bar{x}$ is called a strong solution of Problem 1 . We denote by $S(P 1)$ the set of all such vectors $\bar{x}$.

Problem 2. Find $\bar{x} \in C$ such that for each $y \in C$, there exists $\bar{s} \in T(\bar{x})$ such that

$$
\langle\theta(\bar{x}, \bar{s}), \eta(\bar{x}, y)\rangle \leqslant 0
$$

The solution $\bar{x}$ of this problem is called a weak solution of Problem 1. We denote by $S(P 2)$ the set of all solutions of this problem.

\section{Received 14th April, 1998}

This research was supported by the National Science Council of the Republic of China.

Copyright Clearance Centre, Inc. Serial-fee code: 0004-9729/99 \$A2.00+0.00. 
Problem 3. Find $\bar{x} \in C$ such that

$$
\langle\theta(y, t),(\bar{x}, y)\rangle \leqslant 0, \quad \text { for all } y \in C \text { and } t \in T(y)
$$

We denote by $S(P 3)$ the set of all its solutions.

Inequalities (1), (2) and (3) are known as generalised variational-like inequalities (in short, GVLI). Problem 1 was introduced by Parida and Sen [13] in finite dimensional spaces. They also showed its relation with convex mathematical programming. It was further studied by Yao $[\mathbf{1 9}, \mathbf{2 0}]$ with applications in complementarity problems.

When $\theta(x, s)=s$, for any $x \in C$, Problem 1 was considered by Boss [1], Ding [6] and Siddiqi et al [17].

When $\theta(x, s)=s$ and $\eta(x, y)=x-y$, for any $x, y \in C$ and $s \in T(x)$, the above three problems were studied by Crouzeix [5] in the setting of finite dimensional spaces. In this case, Problem 1 was studied for example by Browder [2], Chowdhury and Tan $[3,4]$, Ding and Tarafdar [7], Fang and Peterson [9], Saigal [14], Shih and Tan [15], Siddiqi and Ansari [16], Tan [18], Yao [21], and Yen [22].

In Section 2, we first prove that $S(P 1)=S(P 2)=S(P 3)$ under certain conditions. Then we define a gap function [10], which provides an optimisation problem formulation, for the generalised variational-like inequality (GVLI)(3). In Section 3, we consider a more general problem which includes Problem 2 as a special case.

Let $C$ and $K$ be nonempty subsets of $E$ and $E^{*}$, respectively. Let $\varphi: K \times C \times C \rightarrow$ $\mathbb{R}$ be a function and $T: C \rightarrow 2^{K}$ be a multifunction. Then we consider the following problem known as a generalised implicit variational problem:

(GIVP) Find $\bar{x} \in C$ such that for each $y \in C$, there exists $\bar{s} \in T(\bar{x})$ such that

$$
\varphi(\bar{s}, \bar{x}, y) \leqslant 0
$$

We prove the existence of its solution by using a result of Chowdhury and Tan [3] which is a generalised form of the Fan-KKM Theorem [8]. As an application, we use our results to prove the existence of solutions of (GVLI).

Let $X, Y$ be subsets of a vector space $E$ such that $\operatorname{co}(\mathrm{X}) \subset Y$. Then the multifunction $F: X \rightarrow 2^{Y}$ is called a KKM-map if for each $A \in \mathcal{F}(X), \operatorname{co}(\mathrm{A}) \subset \bigcup_{x \in A} F(x)$.

The graph of $F$, denoted by $\mathcal{G}(F)$, is

$$
\mathcal{G}(F)=\{(x, y) \in X \times Y: x \in X, y \in F(x)\}
$$

We shall use the following result of Chowdhury and Tan [3] in proving our main results in Section 3. 
THEOREM A. Let $C$ be a nonempty convex set in a topological vector space $E$. Let $G: C \rightarrow 2^{C}$ be a KKM-map such that

(i) $\overline{G\left(y_{0}\right)}$ is compact for some $y_{0} \in C$,

(ii) for each $A \in \mathcal{F}(C)$ with $y_{0} \in A$ and each $y \in \operatorname{co}(\mathrm{A}), G(y) \cap \operatorname{co}(\mathrm{A})$ is closed in $\operatorname{co}(\mathrm{A})$, and

(iii) for each $A \in \mathcal{F}(C)$ with $y_{0} \in A$,

$$
\overline{\left(\bigcap_{y \in \operatorname{co}(\mathrm{A})} G(y)\right)} \cap \operatorname{co}(\mathrm{A})=\left(\bigcap_{y \in \operatorname{co}(\mathrm{A})} G(y)\right) \cap \operatorname{co}(\mathrm{A}) .
$$

Then $\bigcap_{y \in C} G(y) \neq \emptyset$.

The following Kneser minimax theorem [12] will be used in Section 2.

Theorem B. Let $X$ be a nonempty convex subset of a vector space, and let $Y$ be a nonempty compact convex subset of a Hausdorff topological vector space. Suppose that the functional $f: X \times Y \rightarrow \mathbb{R}$ is such that, for each fixed $x \in X, f(x, \cdot)$ is lower semicontinuous and convex, and for each fixed $y \in Y, f(\cdot, y)$ is concave. Then

$$
\min _{y \in Y} \sup _{x \in X} f(x, y)=\sup _{x \in X} \min _{y \in Y} f(x, y) .
$$

\section{A GAP FUNCTION FOR (GVLI)}

Throughout in this paper, unless specified otherwise, $E$ is a topological vector space with dual $E^{*}$.

Let $C$ be a nonempty convex subset of $E$ and $K$ be a nonempty subset of $E^{*}$. Given two functions $\theta: C \times K \rightarrow E^{*}$ and $\eta: C \times C \rightarrow E$, the multifunction $T: C \rightarrow 2^{K}$ is called:

(i) $\eta$-pseudomonotone with respect to $\theta$ if for every pair of points $x \in K$, $y \in K$ and for all $s \in T(x), t \in T(y)$, we have

$$
\langle\theta(x, s), \eta(x, y)\rangle \leqslant 0 \text { implies }\langle\theta(y, t), \eta(x, y)\rangle \leqslant 0 ;
$$

(ii) V-hemicontinuous with respect to $\theta$ and $\eta$ if for all $x, y \in K$, $0<\lambda<1$ and $s_{\lambda} \in T(\lambda y+(1-\lambda) x)$, there exists $s \in T(x)$ such that $\left\langle\theta\left(x, s_{\lambda}\right), \eta(x, y)\right\rangle$ converges to $\langle\theta(x, s), \eta(x, y)\rangle$ as $\lambda$ tends to $0^{+}$.

It is clear that $S(P 1) \subseteq S(P 2)$. By using Theorem B, we prove $S(P 2) \subseteq S(P 1)$.

Proposition 1 . Let $E$ be a Hausdorff topological vector space with dual $E^{*}$ and let $C$ and $K$ be nonempty convex subsets of $E$ and $E^{*}$, respectively. Let $T: C \rightarrow$ $2^{K}$ be a compact convex valued multifunction. Assume that

(a) for each $x, y \in C, s \mapsto\langle\theta(x, s), \eta(x, y)\rangle$ is lower semicontinuous and convex;

(b) for each $x \in K$ and $s \in T(x), y \mapsto\langle\theta(x, s), \eta(x, y)\rangle$ is concave. 
Then $S(P 2) \subseteq S(P 1)$.

Proof: Let $\bar{x} \in C$ be a solution of Problem 2. Then for each $y \in C$, there exists $\bar{s} \in T(\bar{x})$ such that

$$
\langle\theta(\bar{x}, \bar{s}), \eta(\bar{x}, y)\rangle \leqslant 0 .
$$

Define a functional $f: C \times T(\bar{x}) \rightarrow \mathbb{R}$ by

$$
f(y, s)=\langle\theta(\bar{x}, s), \eta(\bar{x}, y)\rangle .
$$

By assumption (a), for each $y \in C$, the functional $s \mapsto f(y, s)$ is lower semicontinuous and convex, and by assumption (b), for each $s \in T(\bar{x})$, the functional $y \mapsto f(y, s)$ is concave. Then by Theorem B, we have

$$
\begin{aligned}
\min _{s \in T(\bar{x})} \sup _{y \in C}\langle\theta(\bar{x}, s), \eta(\bar{x}, y)\rangle & =\sup _{y \in C} \min _{s \in T(\bar{x})}\langle\theta(\bar{x}, s), \eta(\bar{x}, y)\rangle \\
& =\sup _{y \in C}\left[\inf _{s \in T(\bar{x})}\langle\theta(\bar{x}, s), \eta(\bar{x}, y)\rangle\right] \\
& \leqslant 0 .
\end{aligned}
$$

Since $T(\bar{x})$ is compact, there exists a point $\bar{s} \in T(\bar{x})$ such that

$$
\sup _{y \in C}[\langle\theta(\bar{x}, \bar{s}), \eta(\bar{x}, y)\rangle] \leqslant 0
$$

and hence

$$
\langle\theta(\bar{x}, \bar{s}), \eta(\bar{x}, y)\rangle \leqslant 0, \quad \text { for all } y \in C,
$$

that is, $\bar{x} \in S(P 1)$.

Proposition 2. Let $C$ and $K$ be nonempty subsets of $E$ and $E^{*}$, respectively. If $T: C \rightarrow 2^{K}$ is $\eta$-pseudomonotone with respect to $\theta$, then $S(P 1) \subseteq S(P 3)$.

Proposition 3. Let $C$ be a nonempty convex subset of $E$ and $K$ be a nonempty subset of $E^{*}$. Let $\theta(\cdot, \cdot)$ and $\eta(\cdot, \cdot)$ be concave in their first and second arguments, respectively, such that $\eta(x, x)=0$ for all $x \in C$. If $T: C \rightarrow 2^{K}$ is $V$-hemicontinuous with respect to $\theta$ and $\eta$, then $S(P 3) \subseteq S(P 2)$.

Proof: Let $\bar{x} \in S(P 3)$. Then

$$
\langle\theta(y, t), \eta(\bar{x}, y)\rangle \leqslant 0, \quad \text { for all } y \in C \text { and } t \in T(y) .
$$

By the convexity of $C$, for any $\lambda \in(0,1)$, we have

$$
\left\langle\theta\left(\lambda y+(1-\lambda) \bar{x}, s_{\lambda}\right), \eta(\bar{x}, \lambda y+(1-\lambda) \bar{x})\right\rangle \leqslant 0, \quad \text { for all } \quad s_{\lambda} \in T(\lambda y+(1-\lambda) \bar{x}) .
$$


Since $\theta(\cdot, \cdot)$ and $\eta(\cdot, \cdot)$ are concave in their first and second arguments, respectively, and $\eta(x, x)=0$ for all $x \in C$, we have

$$
\begin{aligned}
0 & \geqslant\left\langle\theta\left(\lambda y+(1-\lambda) \bar{x}, s_{\lambda}\right), \eta(\bar{x}, \lambda y+(1-\lambda) \bar{x})\right\rangle \\
& \geqslant \lambda^{2}\left\langle\theta\left(y, s_{\lambda}\right), \eta(\bar{x}, y)\right\rangle+(1-\lambda) \lambda\left\langle\theta\left(\bar{x}, s_{\lambda}\right), \eta(\bar{x}, y)\right\rangle .
\end{aligned}
$$

Dividing by $\lambda>0$, we get

$$
0 \geqslant \lambda\left\langle\theta\left(y, s_{\lambda}\right), \eta(\bar{x}, y)\right\rangle+(1-\lambda)\left\langle\theta\left(\bar{x}, s_{\lambda}\right), \eta(\bar{x}, y)\right\rangle
$$

Taking $\lambda \rightarrow 0^{+}$and by $V$-hemicontinuity with respect to $\theta$ and $\eta$ of $T$, there exists $\bar{s} \in T(\bar{x})$ such that

$$
\langle\theta(\bar{x}, \bar{s}), \eta(\bar{x}, y)\rangle \leqslant 0
$$

and hence $\bar{x} \in S(P 2)$.

By combining Propositions 1-3, we have the following result.

THEOREM 1. Let $E$ be a Hausdorff topological vector space with dual $E^{*}$ and let $C$ and $K$ be nonempty convex subsets of $E$ and $E^{*}$, respectively. Let $T: C \rightarrow 2^{K}$ be compact convex valued, $\eta$-pseudomonotone with respect to $\theta$ and $V$-hemicontinuous with respect to $\theta$ and $\eta$. Let $\theta(\cdot, \cdot)$ and $\eta(\cdot, \cdot)$ be concave in their first and second arguments, respectively, such that $\eta(x, x)=0$ for all $x \in C$. Let $s \mapsto\langle\theta(x, s), \eta(x, y)\rangle$, for all $x, y \in C$, be lower semicontinuous and convex. Then $S(P 1)=S(P 2)=S(P 3)$.

Let $C$ be a nonempty subset of $E$. Then a functional $f: C \rightarrow \mathbb{R} \cup\{-\infty,+\infty\}$ is called a gap function for (GVLI) if

(i) $f(x) \geqslant 0$, for all $x \in C$,

(ii) $f(x)=0$ if and only if $x$ is a solution of (GVLI).

Now, we define a functional $g: C \rightarrow \mathbb{R} \cup\{-\infty,+\infty\}$ as follows:

$$
g(x)=\sup [\langle\theta(y, t), \eta(x, y)\rangle: y \in C \quad \text { and } \quad t \in T(y)]
$$

We also set

$$
m=\inf _{x \in C} g(x) \text { and } M=\{x \in C: g(x)=m\} .
$$

TheOREM 2. Let $C$ be a nonempty subset of $E$ and let $\eta(x, x)=0$ for all $x \in C$. Then $g$ as defined by (5) is a gap function for (GVLI)(3).

Proof: (i) Since $\langle\theta(x, s), \eta(x, x)\rangle=0$ for all $x \in C$ and $s \in T(x)$, we have

$$
g(x) \geqslant 0, \text { for all } x \in C .
$$

(ii) Suppose that $\bar{x} \in C$ is a solution of $(\mathrm{GVLI})(3)$, then

$$
\langle\theta(y, t), \eta(\bar{x}, y)\rangle \leqslant 0, \text { for all } t \in T(y),
$$


and hence

$$
\sup [\langle\theta(y, t), \eta(\bar{x}, y)\rangle: y \in C \quad \text { and } \quad t \in T(y)] \leqslant 0 \text {. }
$$

This implies that $g(\bar{x}) \leqslant 0$. Combining (6) and (7) we get

$$
g(\bar{x})=0
$$

Conversely, let $g(\bar{x})=0$. From (5), we have

$$
g(\bar{x}) \geqslant\langle\theta(y, t), \eta(\bar{x}, y)\rangle, \quad \text { for all } \quad y \in C \text { and } t \in T(y)
$$

and hence

$$
\langle\theta(y, t), \eta(\bar{x}, y)\rangle \leqslant 0, \quad \text { for all } y \in C \text { and } t \in T(y) \text {. }
$$

Therefore, $\bar{x} \in C$ is a solution of (GVLI)(3).

THEOREM 3. Let $C$ be nonempty subset of $E$ and let $\eta(x, x)=0$, for all $x \in C$. If $S(P 3) \neq \emptyset$, then $m=0$ and $M=S(P 3)$.

Proof: Let $S(P 3) \neq \emptyset$. Then from (8), $m=0$.

Let $\bar{x} \in C$ be a solution of (GVLI)(3). Then $g(\bar{x})=0$. But from (6), we have $g(x) \geqslant 0$ for all $x \in C$, and hence $g(\bar{x}) \leqslant g(x)$ for all $x \in C$. Therefore, $\bar{x} \in M$.

Conversely, assume that $\bar{x} \in M$. Then $g(\bar{x})=0$ and thus $\bar{x} \in S(P 3)$. Hence $M=S(P 3)$.

Combining Theorems 1-3, we have the following result.

THEOREM 4. Assume that all the hypotheses of Theorem 1 are satisfied and if $m=0$ and $M \neq \emptyset$, then $M=S(P 1)=S(P 2)=S(P 3)$.

\section{EXISTENCE RESULtS}

We first prove the existence of solution of (GIVP) by using Theorem A.

THEOREM 5. Let $C$ be a nonempty convex subset of $E$ and $K$ be a nonempty subset of $E^{*}$. Let $\varphi: K \times C \times C \rightarrow \mathbb{R}$ be a function and $T: C \rightarrow 2^{K}$ be a multifunction. Assume that

$1^{0}$ for each $A \in \mathcal{F}(C)$ and each $x \in \operatorname{co}(\mathrm{A}), \min _{y \in A} \varphi(s, x, y) \leqslant 0$ for all $s \in$ $T(x)$;

$2^{0}$ for each $A \in \mathcal{F}(C)$ and each $y \in \operatorname{co}(\mathrm{A})$,

$G(y) \cap \operatorname{co}(\mathrm{A})=\{x \in \operatorname{co}(\mathrm{A}):$ there exists $s \in T(x)$ such that $\varphi(s, x, y) \leqslant 0\}$ is closed in $\operatorname{co}(\mathrm{A})$; 
$3^{0}$ for each $A \in \mathcal{F}(C)$ and each $x^{*}, y \in \operatorname{co}(\mathrm{A})$ and for every net $\left\{x_{\alpha}\right\}_{\alpha \in \Gamma}$ in $C$ converging to $x^{*}$, if there exists a net $\left\{s_{\alpha}\right\}$ in $K$ with $s_{\alpha} \in T\left(x_{\alpha}\right)$ for all $\alpha \in \Gamma$, for which

$$
\varphi\left(s_{\alpha}, x_{\alpha}, y\right) \leqslant 0, \text { for all } \alpha \in \Gamma,
$$

then there exists $s^{*} \in T\left(x^{*}\right)$ such that $\varphi\left(s^{*}, x^{*}, y\right) \leqslant 0$;

$4^{0}$ there exists a nonempty closed and compact subset $D$ of $C$ and $z \in D$ such that

$$
\varphi\left(s^{\prime}, x^{\prime}, z\right)>0, \quad \text { for all } x^{\prime} \in C \backslash D \text { and } s^{\prime} \in T\left(x^{\prime}\right) .
$$

Then there exists $\bar{x} \in D$ such that for each $y \in C$, there exists $\bar{s} \in T(\bar{x})$ such that $\varphi(\bar{s}, \bar{x}, y) \leqslant 0$.

Proof: We define the multifunction $G: C \rightarrow 2^{C}$ by $G(y)=\{x \in C:$ there exists $s \in T(x)$ such that $\varphi(s, x, y) \leqslant 0\}$, for each $y \in C$. We show first that $G$ is a KKM-map.

Suppose that $G$ is not a KKM-map. Then for some finite subset $\left\{y_{1}, \ldots, y_{n}\right\}$ of $C$ and $\lambda_{i} \geqslant 0$ for all $i=1, \ldots, n$ with $\sum_{i=1}^{n} \lambda_{i}=1$, we have $x_{0}=\sum_{i=1}^{n} \lambda_{i} y_{i} \notin \bigcup_{i=1}^{n} G\left(y_{i}\right)$. Then, for all $s_{0} \in T\left(x_{0}\right)$,

$$
\varphi\left(s_{0}, x_{0}, y_{i}\right)>0, \quad \text { for all } i=1, \ldots, n
$$

and so

$$
\min _{1 \leqslant i \leqslant n} \varphi\left(s_{0}, x_{0}, y_{i}\right)>0,
$$

which contradicts the assumption $1^{0}$. Hence $G$ is a KKM-map. Moreover, we have,

(i) $G(z) \subset D$ by assumption $4^{0}$, so that $\overline{G(z)} \subset \bar{D}=D$ and hence $\overline{G(z)}$ is compact in $C$;

(ii) for each $A \in \mathcal{F}(C)$ with $z \in A$ and each $y \in \operatorname{co}(\mathrm{A})$,

$G(y) \cap \operatorname{co}(\mathrm{A})=\{x \in \operatorname{co}(\mathrm{A}):$ there exists $s \in T(x)$ such that $\varphi(s, x, y) \leqslant 0\}$ is closed in $\operatorname{co}(\mathrm{A})$ by assumption $2^{0}$.

(iii) for each $A \in \mathcal{F}(C)$ with $z \in A$, if $x^{*} \in \overline{\left(\bigcap_{y \in \operatorname{co}(\mathrm{A})} G(y)\right)} \cap \operatorname{co}(\mathrm{A})$ then $x^{*} \in$ $\overline{\left(\bigcap_{y \in \operatorname{co}(\mathrm{A})} G(y)\right)}$ and $x^{*} \in \operatorname{co}(\mathrm{A})$, and there is a net $\left\{x_{\alpha}\right\}$ in $\bigcap_{y \in \operatorname{co}(\mathrm{A})} G(y)$ such that $x_{\alpha}$ converges to $x^{*}$. For each $y \in \operatorname{co}(\mathrm{A})$, there exists a net $\left\{s_{\alpha}\right\}$ in $K$ with $s_{\alpha} \in T\left(x_{\alpha}\right)$ for which

$$
\varphi\left(s_{\alpha}, x_{\alpha}, y\right) \leqslant 0, \text { for all } \alpha \in \Gamma \text {. }
$$


From assumption $3^{0}$, there exists $s^{*} \in T\left(x^{*}\right)$ such that $\varphi\left(s^{*}, x^{*}, y\right) \leqslant 0$. It follows that $x^{*} \in\left(\bigcap_{y \in \operatorname{co}(\mathrm{A})} G(y)\right) \cap \operatorname{co}(\mathrm{A})$ and hence

$$
\overline{\left(\bigcap_{y \in \operatorname{co}(\mathrm{A})} G(y)\right)} \cap \operatorname{co}(\mathrm{A})=\left(\bigcap_{y \in \operatorname{co}(\mathrm{A})} G(y)\right) \cap \operatorname{co}(\mathrm{A})
$$

By Theorem A, we have $\bigcap_{y \in C} G(y) \neq \emptyset$. Therefore, noting that $\bigcap_{y \in C} G(y) \subseteq G(z) \subseteq$ $D$, there exists $\bar{x} \in D$ such that for each $y \in C$, there exists $\bar{s} \in T(\bar{x})$ such that $\varphi(\bar{s}, \bar{x}, y) \leqslant 0$.

THEOREM 6. Let $C$ be a nonempty convex subset of $E$ and $K$ be a nonempty compact subset of $E^{*}$. Let $\varphi: K \times C \times C \rightarrow \mathbb{R}$ be a function and $T: C \rightarrow 2^{K}$ be a multifunction such that its graph is closed. Assume that

$1^{0}$ for each $A \in \mathcal{F}(C)$ and each $x \in \operatorname{co}(\mathrm{A}), \min _{y \in A} \varphi(s, x, y) \leqslant 0$ for all $s \in$ $T(x)$;

$2^{0}$ for each $A \in \mathcal{F}(C)$ and each $y \in \operatorname{co}(\mathrm{A}), \varphi(\cdot, \cdot, y)$ is lower semicontinuous on $K \times \operatorname{co}(\mathrm{A})$;

$3^{0}$ for each $A \in \mathcal{F}(C)$ and each $x^{*}, y \in \operatorname{co}(\mathrm{A})$ and for every net $\left\{x_{\alpha}\right\}_{\alpha \in \Gamma}$ in $C$ converging to $x^{*}$, if there exists a net $\left\{s_{\alpha}\right\}$ in $K$ with $s_{\alpha} \in T\left(x_{\alpha}\right)$ for all $\alpha \in \Gamma$, for which

$$
\varphi\left(s_{\alpha}, x_{\alpha}, y\right) \leqslant 0 \text { for all } \alpha \in \Gamma
$$

then there exists $x^{*} \in T\left(x^{*}\right)$ such that $\varphi\left(s^{*}, x^{*}, y\right) \leqslant 0$;

$4^{0}$ there exists a nonempty closed and compact subset $D$ of $C$ and $z \in D$ such that

$$
\varphi\left(s^{\prime}, x^{\prime}, z\right)>0, \quad \text { for all } y \in C \backslash D \text { and } s^{\prime} \in T\left(x^{\prime}\right)
$$

Then there exists $\bar{x} \in D$ such that for each $y \in C$, there exists $\bar{s} \in T(\bar{x})$ such that $\varphi(\bar{s}, \bar{x}, y) \leqslant 0$.

Proof: If we prove that for each $A \in \mathcal{F}(C)$ with $z \in A$ and each $y \in \operatorname{co}(\mathrm{A})$,

$$
G(y) \cap \operatorname{co}(\mathrm{A})=\{x \in \operatorname{co}(\mathrm{A}): \text { there exists } s \in T(x) \text { such that } \varphi(s, x, y) \leqslant 0\}
$$

is closed in $\operatorname{co}(\mathrm{A})$ then from Theorem 5 , we get the result.

Indeed, let $\left\{x_{\beta}\right\}_{\beta \in \Lambda}$ be a net in $G(y) \cap \operatorname{co}(\mathrm{A})$ such that $x_{\beta}$ converges to $x$. Then $x \in \operatorname{co}(\mathrm{A})$, because $\operatorname{co}(\mathrm{A})$ is compact (see [3, p.922]). Since $x_{\beta} \in G(y) \cap \operatorname{co}(\mathrm{A})$, there exist $s_{\beta} \in T\left(x_{\beta}\right)$ such that $\varphi\left(s_{\beta}, x_{\beta}, y\right) \leqslant 0$. Since $T(C)$ is contained in a compact set 
$K$, we may assume that $s_{\beta}$ converges to some $s \in K$. Then from the closed graph of $T$. we have $s \in T(x)$. Since $\varphi(\cdot, \cdot, y)$, for each $y \in \operatorname{co}(\mathrm{A})$, is lower semicontinuous, we get

$$
0 \geqslant \liminf _{\beta} \varphi\left(s_{\beta}, x_{\beta}, y\right) \geqslant \varphi(s, x, y)
$$

and hence $x \in G(y) \cap \operatorname{co}(\mathrm{A})$, as desired.

As applications of Theorem 5 and Theorem 6 , we have the following results:

COROLlary 1. Let $C$ be a nonempty convex subset of $E$ and $K$ be a nonempty subset of $E^{*}$. Let $\theta: C \times K \rightarrow E^{*}$ and $\eta: C \times C \rightarrow E$ be functions and $T: C \rightarrow 2^{K}$ be a multifunction. Assume that

$1^{0}$ for each $A \in \mathcal{F}(C)$ and each $x \in \operatorname{co}(\mathrm{A}), \min _{y \in A}\langle\theta(x, s), \eta(x, y)\rangle \leqslant 0$ for all $s \in T(x)$;

$2^{0}$ for each $A \in \mathcal{F}(C)$ and each $y \in \operatorname{co}(\mathrm{A})$, the set

$\{x \in \operatorname{co}(\mathrm{A}):$ there exists $s \in T(x)$ such that $\langle\theta(x, s), \eta(x, y)\rangle \leqslant 0\}$ is closed in $\operatorname{co}(\mathrm{A})$;

$3^{0}$ for each $A \in \mathcal{F}(C)$ and each $x^{*}, y \in \operatorname{co}(\mathrm{A})$ and for every net $\left\{x_{\alpha}\right\}_{\alpha \in \Gamma}$ in $C$ converging to $x^{*}$, if there exists a net $\left\{s_{\alpha}\right\}$ in $K$ with $s_{\alpha} \in T\left(x_{\alpha}\right)$ for all $\alpha \in \Gamma$, for which

$$
\left\langle\theta\left(x_{\alpha}, s_{\alpha}\right), \eta\left(x_{\alpha}, y\right)\right\rangle \leqslant 0, \quad \text { for all } \alpha \in \Gamma,
$$

then there exists $s^{*} \in T\left(x^{*}\right)$ such that $\left\langle\theta\left(x^{*}, s^{*}\right), \eta\left(x^{*}, y\right)\right\rangle \leqslant 0$;

$4^{0}$ there exists a nonempty closed and compact subset $D$ of $C$ and $z \in D$ such that

$$
\left\langle\theta\left(x^{\prime}, s^{\prime}\right), \eta\left(x^{\prime}, z\right)\right\rangle>0, \quad \text { for all } y \in C \backslash D \text { and } s^{\prime} \in T\left(x^{\prime}\right) .
$$

Then there exists $\bar{x} \in D$ such that for each $y \in C$, there exists $\bar{s} \in T(\bar{x})$ such that

$$
\langle\theta(\bar{x}, \bar{s}), \eta(\bar{x}, y)\rangle \leqslant 0 .
$$

Proof: By taking $\varphi(s, x, y)=\langle\theta(x, s), \eta(x, y)\rangle$ in Theorem 5 , we get the result.

Corollary 2. Let $C$ be a nonempty convex subset of $E$ and $K$ be a nonempty compact subset of $E^{*}$. Let $\theta: C \times K \rightarrow E^{*}$ and $\eta: C \times C \rightarrow E$ be functions and $T: C \rightarrow 2^{K}$ be a multifunction such that its graph is closed. Assume that

$1^{0}$ for each $A \in \mathcal{F}(C)$ and each $x \in \operatorname{co}(\mathrm{A}), \min _{y \in A}\langle\theta(x, s), \eta(x, y)\rangle \leqslant 0$ for all $s \in T(x)$; 
$2^{0} \quad$ for each $A \in \mathcal{F}(C)$ and each $y \in \operatorname{co}(\mathrm{A}),\langle\theta(x, s), \eta(x, y)\rangle$ is lower semicontinuous in $(s, x) \in K \times \operatorname{co}(\mathrm{A})$;

$3^{0}$ for each $A \in \mathcal{F}(C)$ and each $x^{*}, y \in \operatorname{co}(\mathrm{A})$ and for every net $\left\{x_{\alpha}\right\}_{\alpha \in \Gamma}$ in $C$ converging to $x^{*}$, if there exists a net $\left\{s_{\alpha}\right\}$ in $K$ with $s_{\alpha} \in T\left(x_{\alpha}\right)$ for all $\alpha \in \Gamma$, for which

$$
\left\langle\theta\left(x_{\alpha}, s_{\alpha}\right), \eta\left(x_{\alpha}, y\right)\right\rangle \leqslant 0, \quad \text { for all } \alpha \in \Gamma \text {, }
$$

then there exists $s^{*} \in T\left(x^{*}\right)$ such that $\left\langle\theta\left(x^{*}, s^{*}\right), \eta\left(x^{*}, y\right)\right\rangle \leqslant 0$;

$4^{0}$ there exists a nonempty closed and compact subset $D$ of $C$ and $z \in D$ such that

$$
\left\langle\theta\left(x^{\prime}, s^{\prime}\right), \eta\left(x^{\prime}, z\right)\right\rangle>0, \quad \text { for all } y \in C \backslash D \text { and } s^{\prime} \in T\left(x^{\prime}\right) .
$$

Then there exists $\bar{x} \in D$ such that for each $y \in C$, there exists $\bar{s} \in T(\bar{x})$ such that

$$
\langle\theta(\bar{x}, \bar{s}), \eta(\bar{x}, y)\rangle \leqslant 0 \text {. }
$$

PRoof: By taking $\varphi(s, x, y)=\langle\theta(x, s), \eta(x, y)\rangle$ in Theorem 6 , we get the result.

Corollary 3. Let $C$ be a nonempty convex subset of $E$ and $K$ be a nonempty compact subset of $E^{*}$. Let $\theta: C \times K \rightarrow E^{*}$ and $\eta: C \times C \rightarrow E$ be functions and $T: C \rightarrow 2^{K}$ be a multifunction such that its graph is closed. Assume that

$1^{0}\langle\theta(x, s), \eta(x, x)\rangle=0$ for all $x \in C$ and $s \in T(x)$;

$2^{0} \quad y \mapsto\langle\theta(x, s), \eta(x, y)\rangle$ is quasiconcave for each fixed $x \in C$ and $s \in T(x)$;

$3^{0}$ for each $A \in \mathcal{F}(C)$ and each $y \in \operatorname{co}(\mathrm{A}),\langle\theta(x, s), \eta(x, y)\rangle$ is lower semicontinuous in $(s, x) \in K \times \operatorname{co}(\mathrm{A})$;

$4^{0}$ for each $A \in \mathcal{F}(C)$ and each $x^{*}, y \in \operatorname{co}(\mathrm{A})$ and for every net $\left\{x_{\alpha}\right\}_{\alpha \in \Gamma}$ in $C$ converging to $x^{*}$, if there exists a net $\left\{s_{\alpha}\right\}$ in $K$ with $s_{\alpha} \in T\left(x_{\alpha}\right)$ for all $\alpha \in \Gamma$, for which

$$
\left\langle\theta\left(x_{\alpha}, s_{\alpha}\right), \eta\left(x_{\alpha}, y\right)\right\rangle \leqslant 0, \quad \text { for all } \alpha \in \Gamma,
$$

then there exists $s^{*} \in T\left(x^{*}\right)$ such that $\left\langle\theta\left(x^{*}, s^{*}\right), \eta\left(x^{*}, y\right)\right\rangle \leqslant 0$;

$5^{0}$ there exists a nonempty closed and compact subset $D$ of $C$ and $z \in D$ such that

$$
\left\langle\theta\left(x^{\prime}, s^{\prime}\right), \eta\left(x^{\prime}, z\right)\right\rangle>0, \quad \text { for all } y \in C \backslash D \text { and } s^{\prime} \in T\left(x^{\prime}\right) \text {. }
$$

Then there exists $\bar{x} \in D$ such that for each $y \in C$, there exists $\bar{s} \in T(\bar{x})$ such that

$$
\langle\theta(\bar{x}, \bar{s}), \eta(\bar{x}, y)\rangle \leqslant 0 \text {. }
$$

PROOF: In view of assumptions $1^{0}$ and $2^{0}$, it is easy to prove that the multifunction $G$ in the proof of Theorem 5 is a KKM-map. By taking $\theta(s, x, y)=$ $\langle\theta(x, s), \eta(x, y)\rangle$ in Corollary 2 , we get the result. 


\section{REFERENCES}

[1] R.K. Boss, 'Ky Fan minimax inequality and non-linear variational inequalities', Nonlinear Anal. Theory, Meth. Appl. 30 (1997), 4161-4170.

[2] F.E. Browder, 'The fixed point theory of multivalued mappings in topological vector spaces', Math. Ann. 177 (1968), 283-301.

[3] M.S.R. Chowdhury and K.K. Tan, 'Generalization of Ky Fan's minimax inequality with applications to generalized variational inequalities for pseudo-monotone operators and fixed point theorems', J. Math. Anal. Appl. 204 (1996), 910-929.

[4] M.S.R. Chowdhury and K.K. Tan, 'Generalized variational inequalites for quasi-monotone operators and applications', Bull. Polish. Acad. Sci. Math. 45 (1997), 25-54.

[5] J.P. Crouzeix, 'Pseudomonotone variational inequality problems: existence of solutions', Math. Programming 78 (1997), 305-314.

[6] X.P. Ding, 'Generalized variational-like inequalities with nonmonotone set-valued mappings', J. Optim. Theory Appl. 95 (1997), 601-613.

[7] X.P. Ding and E. Tarafdar, 'Generalized nonlinear variational inequalities with nonmonotone set-valued mappings', Appl. Math. Lett. 7 (1994), 5-11.

[8] K. Fan, 'A generalization of Tychonoff's fixed point theorem', Math. Ann. 142 (1961), 305-310.

[9] S.C. Fang and E.L. Peterson, 'Generalized variational inequalities', J. Optim. Theory Appl. 38 (1982), 363-383.

[10] D.W. Hearn, 'The gap function of a convex program', Oper. Res. Lett. 1 (1981), 67-71.

[11] C.R. Jou and J.C. Yao, 'Extension of generalized multivalued variational inequalities', Appl. Math. Lett. 6 (1993), 21-25.

[12] H. Kneser, 'Sur un théorème fondamantal de la théorie des jeux', C.R. Acad. Sci. Paris 234 (1952), 2418-2420.

[13] J. Parida and A. Sen, 'A variational-like inequality for multifunctions with applications', J. Math. Anal. Appl. 124 (1987), 73-81.

[14] R. Saigal, 'Extension of the generalized complementarity problems', Math. Oper. Res. 1 (1976), 160-166.

[15] M.H. Shih and K.K. Tan, 'Browder-Hartman-Stampacchia variational inequalities for multi-valued monotone operators', J. Math. Anal. Appl. 134 (1988), 431-440.

[16] A.H. Siddiqi and Q.H. Ansari, 'An iterative method for generalized variational inequalities', Math. Japonica 34 (1989), 475-481.

[17] A.H. Siddiqi, Q.H. Ansari and R. Ahmad, 'On generalized variational-like inequalities', Indian J. Pure Appl. Math. 26 (1995), 1135-1141.

[18] K.K. Tan, 'Comparison theorems on minimax inequalities, variational inequalities, and fixed point theorems', J. London Math. Soc. 28 (1983), 555-562.

[19] J.C. Yao, 'A basic theorem of complementarity for the generalized variational-like inequality problem', J. Math. Anal. Appl. 158 (1991), 124-138.

[20] J.C. Yao, 'Abstract variational inequality problems and a basic theorem of complementarity', Comput. Math. Appl. 25 (1993), 73-79. 
[21] J.C. Yao, 'Multi-valued variational inequalities with $K$-pseudomonotone operators', $J$. Optim. Theory Appl. 83 (1994), 391-403.

[22] C.L. Yen, 'A minimax inequality and its applications to variational inequalities', Pacific J. Math. 97 (1981), $477-481$.

Department of Mathematics Aligarh Muslim University Aligarh - 202002

India
Department of Applied Mathematics

National Sun Yat-sen University

Kaohsiung 804

Taiwan

Republic of China 